\title{
Isolated Colonic Metastasis From Primary Invasive Ductal Breast Carcinoma: Role of Tumor Marker in Early Diagnosis
}

\author{
Srujitha Murukutla ${ }^{\mathrm{a}, \mathrm{d}}$, Abhirami Vivekanandaraja ${ }^{\mathrm{b}}$, Kavitha Paramanathan ${ }^{\mathrm{b}}$, \\ Frank Kong ${ }^{\mathrm{c}}$, Seema Varma ${ }^{\mathrm{b}}$
}

\begin{abstract}
A 59-year-old woman with past medical history significant for invasive ductal carcinoma of the breast successfully treated with right mastectomy with axillary lymph node dissection, radiation, and adjuvant chemotherapy presented with vague abdominal discomfort. She was found to have an elevated CEA level on blood work, which deemed further evaluation. Work up with colonoscopy and biopsy revealed adenocarcinoma of the colon with signet ring cell features consistent with metastatic disease. A primary breast tumor with metastasis to colon is a rare finding. Thus, patients presenting with elevated tumor markers must be judiciously investigated for gastrointestinal tract metastasis.
\end{abstract}

Keywords: Colon metastasis; Immunohistochemistry; Tumor markers; Intraductal carcinoma of the breast

\section{Introduction}

Breast cancer is the leading cause of cancer related mortality worldwide in women. In American women, the lifetime risk of developing breast cancer is one in eight (12\%). According to the American Cancer Society, the estimated number

Manuscript accepted for publication November 17, 2011

\footnotetext{
${ }^{a}$ Staten Island University Hospital, Department of Medicine, 475 Seaview Avenue, Staten Island, New York 10305, USA

${ }^{\mathrm{b}}$ Sanford R Nalitt Institute for Cancer and Blood Related Diseases, Staten Island University Hospital, Division of Hematology and Oncology, Department of Medicine, 256 Mason Avenue, Staten Island, New York 10305, USA

${ }^{\mathrm{c}}$ Staten Island University Hospital, Department of Pathology, 475

Seaview Avenue, Staten Island, New York 10305, USA

${ }^{\mathrm{d}}$ Correspondening author: Srujitha Murukutla.

Email: srujitha.murukutla@gmail.com
}

doi: $10.4021 / \mathrm{jmc} 417 \mathrm{~W}$ of new cases and deaths in women from breast cancer in the United States in 2010 was 207090 and 39840 respectively.

The most common sites of distant metastasis from primary breast cancer are lymph nodes, lung, liver, brain and bone. In rare cases of metastasis to the GI tract, the stomach is most commonly involved site. Metastasis to the duodenum and colon is extremely uncommon (6-18\% to duodenum and $8-12 \%$ to colon) [1].

\section{Case Report}

A 59-year-old woman presented to the ambulatory clinic with symptoms of vague crampy intermittent abdominal pain for 4 weeks not associated with hematochezia, melena, nausea, vomiting or change in bowel habits. Family history was unremarkable. She had a history of Stage $3 \mathrm{C}$ invasive ductal carcinoma of breast (pT3, N3a, Mx) status post right mastectomy with axillary lymph node dissection one year prior to presentation. After surgery, the patient received radiation therapy and 6 courses of adjuvant chemotherapy with dose-dense cyclophosphamide, doxorubicin and paclitaxel. The tumor was positive for estrogen and progesterone receptors and negative for Her-2/neu receptors. Subsequently she

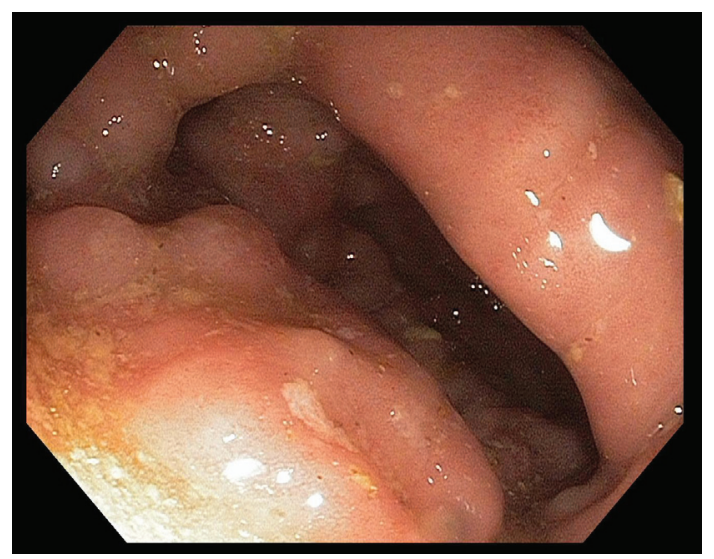

Figure 1. Colonoscopy Image. There are markedly edematous folds in most of the right colon with multiple small superficial erosions. 


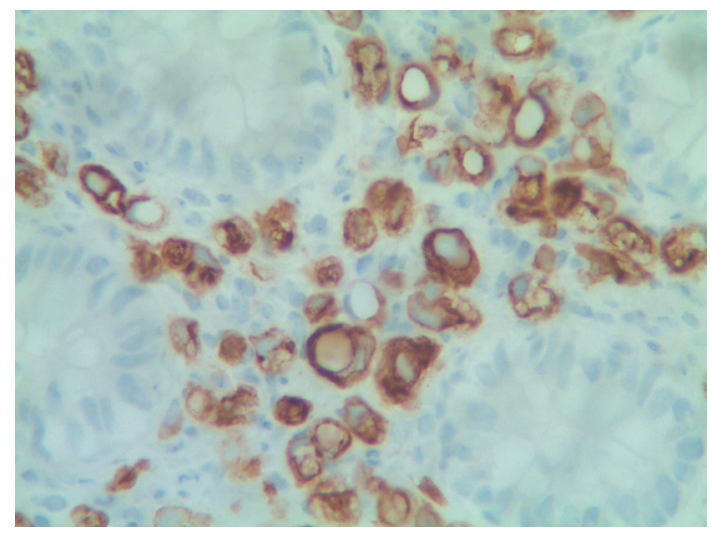

Figure 2. Colonic Biopsy Specimen 1. Immunohistochemical stain (CK7) highlights the metastatic carcinoma cells in the stroma of colonic mucosa (IHC, x 40).

was prescribed hormonal therapy with anastrozole, which the patient was taking for the last 6 months prior to this presentation. She had a surveillance PET/CT study 3 months prior to presentation, which did not show any evidence of recurrence.

Vital signs on evaluation were as follows: blood pressure: 103/68 $\mathrm{mm}$ of $\mathrm{Hg}$, pulse rate: 84 per minute, respiratory rate: 16 per minute and temperature: 98 degree Fahrenheit. Breast examination did not reveal any masses; skin was normal except for surgical scars from the prior mastectomy. Abdominal examination did not reveal any masses or organomegaly. Rectal examination revealed normal tone, no mass and no gross blood. Fecal occult blood test was negative. Hemogram, renal and liver function tests were within normal limits. Tumor markers were as follows: CA27-29: 112.13 units per milliliter, CA15-3: 67.1 units per milliliter and CEA: 690 nanograms per milliliter. Considering her age, symptoms and elevated tumor markers a colonoscopy was performed that revealed markedly edematous folds in the right colon with multiple superficial erosions, morphologically resembling ischemic colitis (Fig. 1). Pathology of the biopsy specimen showed poorly differentiated adenocarcinoma with signet ring cell features. Immunohistochemical stains were positive for cytokeratin 7, mammoglobin, GCDFP and CA-125 and negative for cytokeratin 20 and TTF, which was consistent with a primary breast neoplasm (Fig. 2, 3).

\section{Discussion}

The incidence of metastases to the GI tract from a primary breast cancer is extremely rare. Sites of gastrointestinal metastasis included esophagus (8\%), stomach $(28 \%)$, small intestine (19\%), and colon and rectum (45\%). Infiltrating lobular breast cancer has an increased predilection to involve the GI tract relative to ductal carcinoma. Borst et al investigated



Figure 3. Colonic Biopsy Specimen 2. Immunohistochemical stain (GCDFP15) shows a few tumor cells react to antibody of Gross Cystic Disease Fluid Protein 15, which is a breast tissue marker, consistent with metastatic tumor of breast primary (IHC, x 40).

2600 cases of invasive lobular and invasive ductal breast cancer and respective metastatic patterns over 18 years. The study found that only 17 patients in the study population had GI tract involvement, with a greater prevalence among patients with lobular carcinoma [2]. Lobular carcinoma is also known to cause linitis plastica type lesions in the colon and stomach [3].

Metastasis to the GI tract can have a wide range of clinical manifestations. Patients may be asymptomatic, present with symptoms of bowel obstruction, abdominal mass, anemia, and inflammatory bowel disease or mimic a primary neoplasm [3-13]. Abdominal symptoms can also occur as a side effect of chemotherapeutic agents and can often obscure necessary detailed work-up. For example, docetaxel-based chemotherapy in patients with metastatic breast cancer is known to be associated with colitis [14]. Colorectal involvement usually presents with diarrhea, abdominal pain, abdominal mass or obstruction. However, in this case, the patient had non-specific abdominal pain and the elevated CEA level was an isolated marker for intestinal metastasis. The median time from diagnosis of primary breast cancer to colonic metastasis is 6 to 8 years but there are reports of cases diagnosed as early as 4 months and as late as 28 years after initial diagnosis (Table 1). Therefore, a high index of suspicion is required in patients with even subtle GI symptoms in order to exclude metastasis to the GI tract.

Tumor markers play an important role in the early diagnosis of disease recurrence and may be the only abnormality [15]. Molina $\mathrm{R}$ et al evaluated 250 patients with primary breast cancer without evidence of residual disease. They found tumor marker elevation as the first sign of relapsing disease in $69.5 \%$ of patients. It should be noted however, that neither the National comprehensive cancer network nor the American society of clinical oncology recommend utilizing tumors markers in routine staging, 


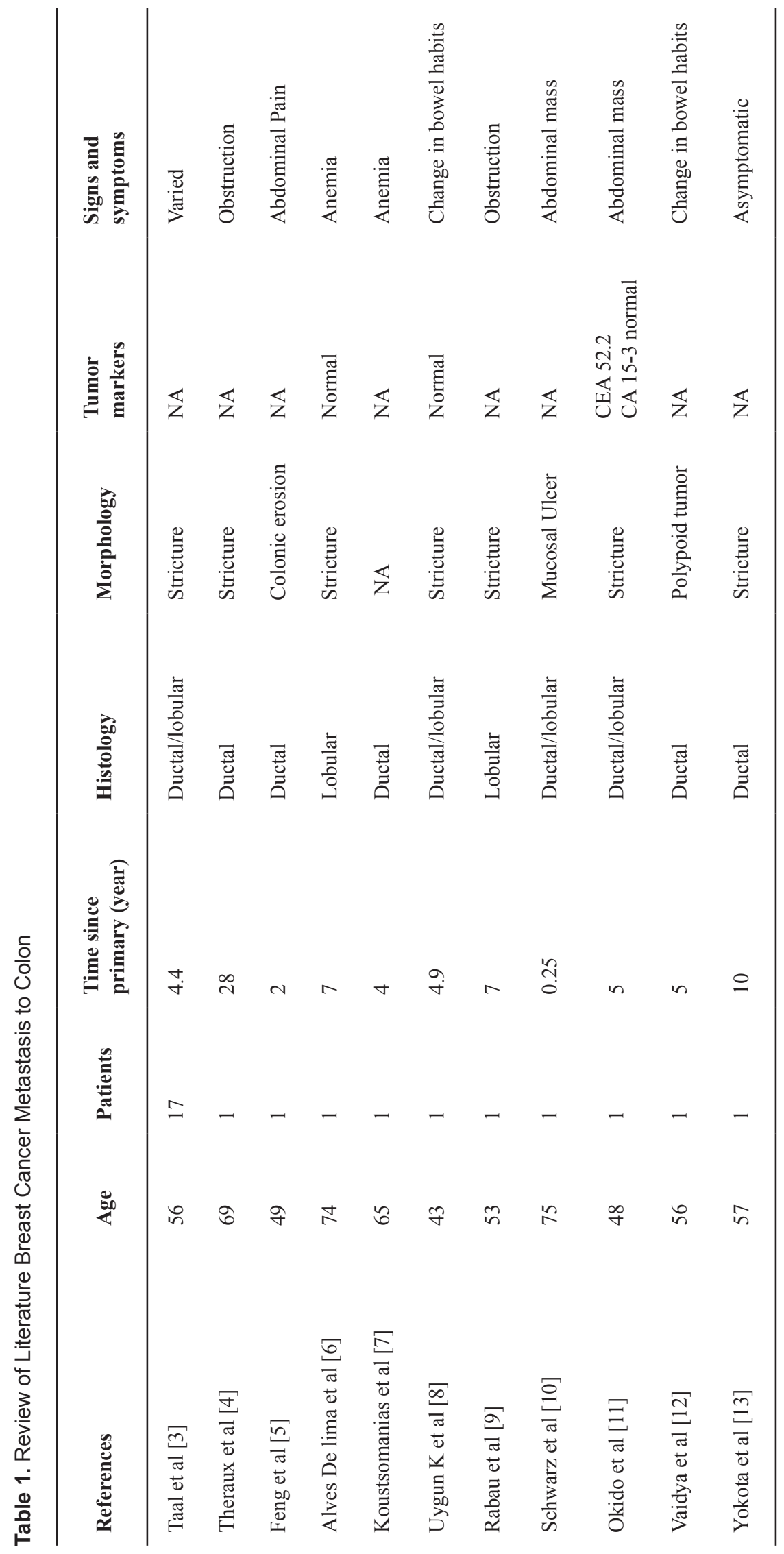


diagnosis or surveillance of breast cancer patients.

A patient with breast cancer presenting with gastrointestinal symptoms should be further investigated with endoscopy and deep tissue biopsies. Endoscopic findings can be very non-specific. Due to predominant sub-mucosal or serosal involvement, results may not show any mucosal abnormalities [3]. Morphologically it may be difficult to differentiate metastasis from a primary neoplasm versus other benign conditions. In our patient, there was no definite polyp, tumor or ulcer identified on colonoscopy. The lesion only showed superficial erosions with increased friability. Therefore, even a benign appearance warrants further evaluation with deep tissue biopsies.

Second primary neoplasms are more common than metastasis to GI tract in breast cancer patients. Thus, it is important to perform immunohistochemical analysis and histological comparison with the previous breast specimen. Such an approach would avoid unnecessary surgical procedures and may guide appropriate treatment strategies. Park et al looked at immunohistochemical markers in 314 adenocarcinomas of different origins and found the following tumor markers specific for breast origin: GCDFP-15+, TTF-1-, CDX2-, CK7+, CK20- or ER+, TTF-1-, CDX2-, CK20, CEA-, MU$\mathrm{C} 5 \mathrm{AC}$ [16]. In our patient, the immunohistochemistry was positive for cytokeratin 7, GCDFP and negative for cytokeratin 20, which is specific for breast. We concluded that our patient presented with symptoms secondary to GI tract metastases from a primary invasive ductal carcinoma.

\section{Treatment}

Although there is no consensus on treatment of GI tract metastases from a primary breast carcinoma, systemic chemotherapy and hormonal therapy have been recommended. Mclemore et al examined 73 patients with gastrointestinal metastasis, carcinomatosis or both and found no survival benefit in patients who had surgical intervention. Although not statistically significant, there was improved survival in patients who had palliative surgery (44 versus 9 months). Thus palliative surgery may have a role in patients presenting with obstruction and/or perforation [17].

\section{Conclusion}

It can be very difficult to diagnose colorectal metastasis due to non-specific symptoms with a variable clinical presentation. In this case, we emphasize the importance of performing a thorough gastrointestinal work-up in patients with a history of breast cancer presenting with vague abdominal symptoms and tumor marker elevation. Endoscopy and deep tissue biopsies should be performed with appropriate immunohistochemical analysis even if the lesions appear benign. Results should be interpreted in conjunction with all prior diagnoses.
To the best of our knowledge, this is the first case of colonic metastasis from breast cancer diagnosed by an elevated CEA level, which is a marker of primary colorectal carcinoma to be reported in the literature.

\section{Conflict of Interest}

None to declare.

\section{Sources of Support}

None to declare.

\section{Disclaimer}

None.

\section{References}

1. Cifuentes N, Pickren JW. Metastases from carcinoma of mammary gland: an autopsy study. J Surg Oncol. 1979;11(3):193-205.

2. Borst MJ, Ingold JA. Metastatic patterns of invasive lobular versus invasive ductal carcinoma of the breast. Surgery. 1993;114(4):637-641; discussion 641-632.

3. Taal BG, den Hartog Jager FC, Steinmetz R, Peterse H. The spectrum of gastrointestinal metastases of breast carcinoma: II. The colon and rectum. Gastrointest Endosc. 1992;38(2):136-141.

4. Theraux J, Bretagnol F, Guedj N, Cazals-Hatem D, Panis Y. Colorectal breast carcinoma metastasis diagnosed as an obstructive colonic primary tumor. A case report and review of the literature. Gastroenterol Clin Biol. 2009;33(12):1114-1117.

5. Feng CL, Chou JW, Huang SF. Colonic metastasis from carcinoma of the breast presenting with colonic erosion. Endoscopy. 2009;41 Suppl 2:E276-277.

6. Alves de Lima DC, Alberti LR. Breast cancer metastasis to the colon. Endoscopy. 2011;43 Suppl 2 UCTN:E143-144.

7. Koutsomanis D, Renier JF, Ollivier R, Moran A, el-Haite AA. Colonic metastasis of breast carcinoma. Hepatogastroenterology. 2000;47(33):681-682.

8. Uygun K, Kocak Z, Altaner S, Cicin I, Tokatli F, Uzal C. Colonic metastasis from carcinoma of the breast that mimics a primary intestinal cancer. Yonsei Med J. 2006;47(4):578-582.

9. Rabau MY, Alon RJ, Werbin N, Yossipov Y. Colonic metastases from lobular carcinoma of the breast. Report of a case. Dis Colon Rectum. 1988;31(5):401-402. 
10. Schwarz RE, Klimstra DS, Turnbull AD. Metastatic breast cancer masquerading as gastrointestinal primary. Am J Gastroenterol. 1998;93(1):111-114.

11. Okido M, Seo M, Hamada Y, Kurihara S, Matsumoto K, Konomi H, Kato M, et al. Metastatic breast carcinoma simulating linitis plastica of the colon: report of a case. Surg Today. 2011;41(4):542-545.

12. Vaidya JS, Mukhtar H, Bryan R. Colonic metastasis from a breast cancer--a case report and a few questions. Eur J Surg Oncol. 2002;28(4):463-464.

13. Yokota T, Kunii Y, Kagami M, Yamada Y, Takahashi M, Kikuchi S, Nakamura M, et al. Metastatic breast carcinoma masquerading as primary colon cancer. Am J Gastroenterol. 2000;95(10):3014-3016.

14. Kreis W, Petrylak D, Savarese D, Budman D. Coli- tis and docetaxel-based chemotherapy. Lancet. 2000;355(9221):2164.

15. Molina R, Jo J, Filella X, Zanon G, Farrus B, Munoz M, Latre ML, et al. C-erbB-2, CEA and CA 15.3 serum levels in the early diagnosis of recurrence of breast cancer patients. Anticancer Res. 1999;19(4A):2551-2555.

16. Park SY, Kim BH, Kim JH, Lee S, Kang GH. Panels of immunohistochemical markers help determine primary sites of metastatic adenocarcinoma. Arch Pathol Lab Med. 2007;131(10):1561-1567.

17. McLemore EC, Pockaj BA, Reynolds C, Gray RJ, Hernandez JL, Grant CS, Donohue JH. Breast cancer: presentation and intervention in women with gastrointestinal metastasis and carcinomatosis. Ann Surg Oncol. 2005;12(11):886-894. 